\title{
Allongement de l'intervalle QT causé par des médicaments chez l'enfant : comment vont les enfants?
}

\author{
par James E. Tisdale
}

L es torsades de pointes (TdP) sont une forme de tachycardie

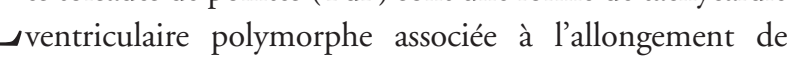
l'intervalle QT corrigé pour la fréquence cardiaque (QTc) sur un électrocardiogramme $(\mathrm{ECG})^{1}$. Les TdP peuvent être catastrophiques, car elles peuvent mener à la mort subite cardiaque ${ }^{1}$. Lallongement de l'intervalle QTc est un trouble héréditaire ou acquis. La forme héréditaire signifie le syndrome du QT long congénital dont il existe au moins quinze types ${ }^{2}$, alors que la forme acquise est plus souvent causée par des médicaments ${ }^{3}$. Au cours des trois dernières décennies, plusieurs médicaments, notamment l'astémizole, le cisapride, la grépafloxacine, la prénylamine et la terfénadine, ont été retirés du marché canadien à cause de décès attribuables aux $\mathrm{TdP}^{4}$. Néanmoins, plus de 100 médicaments ayant le potentiel d'allonger l'intervalle QTc et d'entraîner des TdP demeurent disponibles 5 .

Bien que la plupart des cliniciens savent que des médicaments peuvent causer l'allongement de l'intervalle QTc et l'apparition de TdP chez l'adulte, bon nombre n'ont pas autant conscience que ces effets indésirables liés à un médicament peuvent aussi se produire chez l'enfant. Or, on a publié de nombreux cas de TdP d'origine médicamenteuse chez l'enfant ${ }^{6}$. Des cas de TdP chez l'enfant ont été associés à différents médicaments, notamment l'astémizole, le cisapride, la pentamidine, la rispéridone, des antiarythmiques (l'amiodarone, la procainamide et le sotalol) et des antifongiques (le fluconazole et le voriconazole) ${ }^{6}$.

Dans le présent numéro du Journal canadien de la pharmacie hospitalière, Morris et collab. ${ }^{7}$ présentent une revue de la littérature sur l'allongement de l'intervalle QT associé à la dompéridone chez des enfants qui ne sont pas atteints d'un cancer. La dompéridone est l'agent procinétique gastro-intestinal de choix pour traiter le reflux gastro-œsophagien qui survient chez l'enfant, normalement à l'âge de 5 à 18 mois ${ }^{7}$. Les auteurs décrivent les résultats de trois études de cohorte prospectives, d'un essai clinique à répartition aléatoire et d'une observation clinique sur l'utilisation de dompéridone chez un total de 137 enfants âgés entre deux jours et neuf mois ${ }^{7}$. Il n'y a eu aucun cas d'allongement de l'intervalle QTc chez la plupart d'entre eux, mais pour six patients l'intervalle QTc était anormalement long ( $\geq 450 \mathrm{~ms}$ ) et trois d'entre eux affichaient un intervalle QTc de plus de $460 \mathrm{~ms}$, allant jusqu'à un maximum de $490 \mathrm{~ms}^{8}$. Dans l'une des études prospectives, la dompéridone était associée à un allongement significatif de l'intervalle QTc moyen comparativement aux valeurs précédant le traitement chez des nouveau-nés ${ }^{9}$. Bien qu'aucun patient apparaissant dans cette revue de la littérature n'ait affiché un intervalle QTc de plus de $500 \mathrm{~ms}$ ou une augmentation de l'intervalle QTc de plus de $60 \mathrm{~ms}$ en comparaison avec la valeur avant traitement (les seuils généralement acceptés pour une augmentation marquée du risque de $\mathrm{TdP}^{6}$ ), l'allongement par un médicament de l'intervalle QTc à une valeur plus élevée que $450 \mathrm{~ms}$ est préoccupant. Heureusement, aucun patient dans cette analyse n'a éprouvé de TdP ou n'a été victime de mort subite cardiaque.

Le taux d'incidence global de TdP causées par des médicaments n'est pas bien décrit chez l'adulte et est généralement inconnu chez l'enfant. L'incidence de cas de TdP associées à différents médicaments (calculée chez des populations composées presque exclusivement d'adultes) varie de $2 \%$ à $12 \%$ selon le médicament, la dose et d'autres facteurs de risque ${ }^{3}$. Dans un rapport étudié par Morris et collab. ', l'incidence de l'allongement de l'intervalle QTc associé à la dompéridone chez le nourrisson était de 4,4\% ${ }^{10}$.

Les TdP et l'allongement de l'intervalle QTc causés par des médicaments dépendent beaucoup de facteurs prédisposants et ne se produisent que rarement chez des patients ne présentant aucun facteur de risque concomitant ${ }^{6}$. Chez l'adulte, on compte comme facteurs de risque : le sexe féminin; l'âge avancé (plus de 65 ans); l'hypokaliémie, l'hypomagnésémie ou l'hypocalcémie; l'insuffisance cardiaque avec fraction d'éjection réduite; la bradycardie; un traitement avec plus d'un médicament allongeant l'intervalle QTc; et des phénomènes qui augmentent les concentrations plasmatiques de tels médicaments, comme des maladies 
rénales ou hépatiques, des interactions médicamenteuses et une administration par voie intraveineuse rapide ${ }^{6}$. On ignore lesquels de ces facteurs de risque (hormis l'âge avancé) interviennent aussi chez l'enfant. Dans une petite étude $(n=31)$, les facteurs de risque indépendants pour l'allongement de l'intervalle QTc associé à la dompéridone chez le nouveau-né étaient l'âge gestationnel avancé et, fait paradoxal, des taux sériques de potassium à la limite supérieurs de la normale 9 . On ne sait pas pourquoi l'allongement est associé à des taux sériques de potassium à la limite supérieure de la normale plutôt qu'à l'hypokaliémie, le facteur de risque largement reconnu. Compte tenu de ce que l'on connaît des effets sur les canaux potassiques, on pourrait croire que l'hypokaliémie, l'hypomagnésémie, la bradycardie et des concentrations plasmatiques élevées de médicaments allongeant l'intervalle QTc sont des facteurs de risque chez l'enfant comme c'est le cas chez l'adulte $^{6}$. Cependant, le sexe féminin n'est pas un facteur de risque d'allongement de l'intervalle QTc et de TdP chez l'enfant prépubère. Les données probantes montrent que l'intervalle QTc est semblable chez les garçons et les filles jusqu'au début de la puberté, après quoi les intervalles QTc divergent ${ }^{11}$. Cette différence est probablement causée par la production accrue de testostérone chez les garçons à la puberté, phénomène dont on a montré qu'il est associé à un intervalle QTc plus court ${ }^{6}$. Ainsi, le sexe féminin n'est un facteur de risque d'allongement de l'intervalle QTc et de TdP qu'à partir de la puberté.

Pour les patients adultes hospitalisés recevant un médicament qui cause l'allongement de l'intervalle QTc, on recommande de surveiller continuellement l'intervalle QTc et de maintenir des taux sériques normaux de potassium, de magnésium et de calcium ${ }^{1}$. Si l'intervalle QTc d'un patient dépasse $500 \mathrm{~ms}$ ou surpasse de plus de $60 \mathrm{~ms}$ la valeur mesurée avant le traitement, on recommande : d'employer, si possible, un traitement qui n'allonge pas l'intervalle QTc; d'évaluer la possibilité d'interactions médicamenteuses, de bradyarythmies ou de désordres électrolytiques; et de se préparer à prendre en charge les TdP, le cas échéant ${ }^{1}$. Aucune recommandation pour les enfants ou les nouveau-nés hospitalisés qui reçoivent une pharmacothérapie allongeant l'intervalle QTc n'a été publiée à ce jour, mais il serait raisonnable d'adopter des recommandations semblables à celles destinées aux adultes. Chez l'enfant présentant des facteurs de risque de TdP, mais pour qui il est impossible de surveiller continuellement l'intervalle QTc, un ECG à 12 dérivations doit être réalisé au début du traitement, puis 3 à 7 jours après avoir amorcé la pharmacothérapie causant l'allongement de l'intervalle QTc.

La revue réalisée par Morris et collab. ${ }^{7}$ nous rappelle que l'allongement de l'intervalle QTc causé par des médicaments ne se limite pas à l'adulte, mais que l'enfant et le nouveau-né y sont aussi sujets, particulièrement s'ils présentent des facteurs prédisposants. Une surveillance adéquate de l'intervalle QTc et une attention aux facteurs de risque modifiables sont des gestes importants pour réduire la possibilité d'allongement de l'intervalle QTc et de TdP chez l'enfant et le nouveau-né qui reçoivent une pharmacothérapie allongeant l'intervalle QTc.

[Traduction par l'éditeur]

\section{Références}

1. Drew BJ, Ackerman MJ, Funk M, Gibler WB, Kligfield P, Menon V, et collab.; American Heart Association Acute Cardiac Care Committee of the Council on Clinical Cardiology, the Council on Cardiovascular Nursing, and the American College of Cardiology Foundation. Prevention of torsade de pointes in hospital settings: a scientific statement from the American Heart Association and the American College of Cardiology Foundation. Circulation. 2010;121(8):1047-60. Erratum publié dans Circulation. 2010; 122(8):e440.

2. Mizusawa Y, Horie M, Wilde AAM. Genetic and clinical advances in congenital long QT syndrome. Circ J. 2014;78(12):2827-33.

3. Tisdale JE. Drug-induced QT interval prolongation and torsades de pointes: role of the pharmacist in risk assessment, prevention and management. Can Pharm J. 2016;149(3):139-52.

4. Lexchin J. Drug withdrawals from the Canadian market for safety reasons, 1963-2004. CMAJ. 2005;172(6):765-7.

5. Woosley RL, Romero KA. QTDrugs Lists [base de données]. Oro Valley (AZ) : AZCERT, Inc. Publié au : https://www.crediblemeds.org/index.php/ login/dlcheck. Consulté le 20 avril 2016.

6. Tisdale JE. Ventricular arrhythmias. Dans : Tisdale JE, Miller DA, editors. Drug-induced diseases. Prevention, detection and management. 2e édition. Bethesda (MD) : American Society of Health-Systems Pharmacists; 2010. p. 485-515.

7. Morris AD, Chen J, Lau E, Poh J. Domperidone-associated QT interval prolongation in non-oncologic pediatric patients: a review of the literature. Can J Hosp Pharm. 2016;69(3):224-30.

8. Günlemez A, Babaoglu A, Arisoy AE, Türker G, Gökalp AS. Effect of domperidone on the QTc interval in premature infants. J Perinatol. 2010; 30(1):50-3.

9. Djeddi D, Kongolo G, Lefaix C, Mounard J, Léké A. Effect of domperidone on QT interval in neonates. J Pediatr. 2008;153(5):663-6.

10. Vieira MC, Miyague NI, Van Steen K, Salvatore S, Vandenplas Y. Effects of domperidone on QTc interval in infants. Acta Paediatr. 2012;101(5):494-6.

11. Rautaharju PM, Zhou SH, Wong S, Calhoun HP, Berenson GS, Prineas R, et collab. Sex differences in the evolution of the electrocardiographic QT interval with age. Can J Cardiol. 1992;8(7):690-5.

James E Tisdale, B. Sc. Pharm., Pharm. D, est professeur titulaire, Département de pratique pharmaceutique de la Faculté de pharmacie de l'Université Purdue et professeur associé de l'École de médecine de I'Université d'Indiana, à Indianapolis, en Indiana, aux États-Unis. II est également rédacteur adjoint au Journal canadien de la pharmacie hospitalière.

Intérêts concurrents : James Tisdale est membre bénévole de la Commission consultative de la base de données QTDrugs Lists pour le site Web www.crediblemeds.org (qui est cité à la référence 5 du présent éditorial). L'auteur n'a déclaré aucun autre intérêt concurrent.

\section{Adresse de correspondance :}

Dr James E. Tisdale

Department of Pharmacy Practice

College of Pharmacy

Purdue University

640 Eskenazi Avenue

Indianapolis IN 46202 USA

Courriel : jtisdale@purdue.edu 